\title{
In vivo reorganization of the actin cytoskeleton in leaves of Nicotiana tabacum L. transformed with plastin-GFP. Correlation with light-activated chloroplast responses
}

\author{
Anna Anielska-Mazur1, Tytus Bernaś ${ }^{2}$ and Halina Gabrys*1
}

\begin{abstract}
Address: ${ }^{1}$ Department of Plant Physiology and Biochemistry, Faculty of Biochemistry, Biophysics and Biotechnology, Jagiellonian University, Gronostajowa 7, 30-387 Kraków, Poland and 2Department of Plant Anatomy and Cytology, Faculty of Biology and Environmental Protection, Silesian University, Jagiellońska 26/28, 40-032 Katowice, Poland

Email: Anna Anielska-Mazur - aam@ibb.waw.pl; Tytus Bernaś- tbernas@us.edu.pl; Halina Gabrys* - halina.gabrys@uj.edu.pl

* Corresponding author
\end{abstract}

Published: 29 May 2009

BMC Plant Biology 2009, 9:64 doi:10.1 I86//47/-2229-9-64
Received: 3 April 2009

Accepted: 29 May 2009

This article is available from: http://www.biomedcentral.com/I47/-2229/9/64

(C) 2009 Anielska-Mazur et al; licensee BioMed Central Ltd.

This is an Open Access article distributed under the terms of the Creative Commons Attribution License (http://creativecommons.org/licenses/by/2.0), which permits unrestricted use, distribution, and reproduction in any medium, provided the original work is properly cited.

\begin{abstract}
Background: The actin cytoskeleton is involved in the responses of plants to environmental signals. Actin bundles play the role of tracks in chloroplast movements activated by light. Chloroplasts redistribute in response to blue light in the mesophyll cells of Nicotiana tabacum. The aim of this work was to study the relationship between chloroplast responses and the organization of actin cytoskeleton in living tobacco cells. Chloroplast movements were measured photometrically as changes in light transmission through the leaves. The actin cytoskeleton, labeled with plastin-GFP, was visualised by confocal microscopy.

Results: The actin cytoskeleton was affected by strong blue and red light. No blue light specific actin reorganization was detected. EGTA and trifluoperazine strongly inhibited chloroplast responses and disrupted the integrity of the cytoskeleton. This disruption was reversible by $\mathrm{Ca}^{2+}$ or $\mathrm{Mg}^{2+}$. Additionally, the effect of trifluoperazine was reversible by light. Wortmannin, an inhibitor of phosphoinositide kinases, potently inhibited chloroplast responses but did not influence the actin cytoskeleton at the same concentration. Also this inhibition was reversed by $\mathrm{Ca}^{2+}$ and $\mathrm{Mg}^{2+}$. Magnesium ions were equally or more effective than $\mathrm{Ca}^{2+}$ in restoring chloroplast motility after treatment with EGTA, trifluoperazine or wortmannin.

Conclusion: The architecture of the actin cytoskeleton in the mesophyll of tobacco is significantly modulated by strong light. This modulation does not affect the direction of chloroplast redistribution in the cell. Calcium ions have multiple functions in the mechanism of the movements. Our results suggest also that $\mathrm{Mg}^{2+}$ is a regulatory molecule cooperating with $\mathrm{Ca}^{2+}$ in the signaling pathway of blue light-induced tobacco chloroplast movements.
\end{abstract}

\section{Background}

Actin cytoskeleton (AC) provides tracks for myosin-mediated movements of organelles in plant cells [1]. The dynamic nature of the cytoskeleton depends on actin- binding proteins which control the assembly of actin filaments (AFs) and their organization into higher-order structures [2]. 
On the basis of AC, chloroplasts change their intracellular arrangement in response to light. These movements are controlled only by blue light in higher plants [3]. Weak blue light (wBL) induces an accumulation response in which chloroplasts gather along the cell walls perpendicular to the light direction. Strong blue light (SBL) induces an avoidance response in which they stay at the walls parallel to the light direction, away from the most illuminated parts of the cell. The light signal is perceived by phototropins (phot1 and phot2), blue-light photoreceptors localised at the plasma membrane $[4,5]$. Both phototropins mediate chloroplast accumulation, whereas phot2 mediates the avoidance response [6,7]. Chloroplasts of several algae, mosses, ferns and aquatic angiosperms respond also to red light $[8,9]$.

Chloroplasts move along AFs using myosins associated with their membrane [10-12]. Microtubules do not seem to be involved in the directional redistribution of chloroplasts in higher land plants $[1,13]$. In spite of recent advances little is known about the pathway upon which the blue light signal is transmitted from phototropins to the motor apparatus (see reviews $[14,15]$ ). Only two types of secondary messengers have been critically discussed in this context: $\mathrm{Ca}^{2+}$ ions and the phosphoinositide kinases.

Calcium ions regulate the activity of many cytoskeletal proteins and act as secondary messenger in several plant signalling pathways including those initiated by phototropins [16-19]. As shown in studies employing the aequorin $\mathrm{Ca}^{2+}$ reporter system, BL acting through phot1 induced an increase in cytosolic $\mathrm{Ca}^{2+}$ in Arabidopsis and tobacco seedlings [20]. Phototropin 1 was also responsible for triggering an influx of $\mathrm{Ca}^{2+}$ across the plasma membrane in Arabidopsis seedling hypocotyls [21] and for activating $\mathrm{Ca}^{2+}$ channels at the plasma membrane of Arabidopsis mesophyll protoplasts [22]. Calcium ions have been postulated as a potential secondary messenger in red light-controlled chloroplast movements and cytoplasmic streaming in the aquatic angiosperm, Vallisneria gigantea [23]. The function of $\mathrm{Ca}^{2+}$ in BL-induced movements still awaits clarification. Manipulating cytosolic calcium homeostasis with various calcium antagonists was shown to interfere with both wBL and SBL chloroplast responses $[24,25]$. However, this does not explain the role calcium ions play in their mechanisms.

A second category of potential secondary messengers has been proposed, based on different effects of wortmannin, an inhibitor of phosphoinositide-3-kinases, on accumulation and avoidance chloroplast responses in the duckweed Lemna trisulca [26]. The authors put forward a model linking the phosphoinositide kinases and other phosphoinositide cycle enzymes with light signal transduction.
According to this model the direction of chloroplast movements is determined by phosphoinositides, whereas $\mathrm{Ca}^{2+}$ ions are required only to control the activity of the motor apparatus.

In the last few years we have sought a target of the phototropin-mediated signal which initiates the chloroplast redistribution. In the red-sensitive species actin cytoskeleton was shown to play that role for the phytochromemediated signaling $[8,9]$. Our recent results point to myosin rather than to actin as the target in blue-sensitive higher plants $[12,27]$. Up till now, the light effects on AC were studied using fixed tissue. Here, we attempted to visualize the cytoskeleton in living mesophyll cells. The truncated plastin-GFP construct [28] was successfully expressed in mature tobacco leaves giving a stable, fully functional transgenic line. The objective of the present study was to perform life imaging of the actin dynamics in this transgenic Nicotiana tabacum system, and to take a step toward identifying secondary messengers and relations between them in blue light-controlled chloroplast movements. To achieve the latter goal we compared the effects of calcium agonists/antagonists and of wortmannin on $\mathrm{AC}$ and on the chloroplast responses.

\section{Results \\ Characteristics of the transgenic tobacco line}

The expression of plastin-GFP did not affect the responses of chloroplasts in Nicotiana tabacum. The amplitudes and kinetics of these responses were about the same in both transformed and non-transformed three-month-old plants (Fig. 1A: a, b). Notably, the chloroplast redistribution was much weaker in younger plants grown in vitro for up to two months after each passage (Fig. 1A: c, d). Instead of filamentous structures present in three-monthold plants, fluorescent speckles and diffuse fluorescence were observed throughout the young tissue (Fig. 1B). Obviously, the actin tracks necessary for chloroplast movements were undeveloped in young plants.

The expression of plastin-GFP was generally low, with varying levels in different cells and tissues. Two plant generations were screened for the most uniform expression of plastin-GFP in the spongy mesophyll cells. The best plant was reproduced vegetatively and used in further investigations. The varied expression of plastin-GFP between plants coming from different generations, observed in the confocal images at the protein level, was confirmed by RTPCR at the level of mRNA (Fig. 1C). No differences in germination, development and flowering were found between transgenic and wild type plants. Also the efficiency of photosynthesis measured as in vivo chlorophyll fluorescence was identical in the two groups (results not shown). 

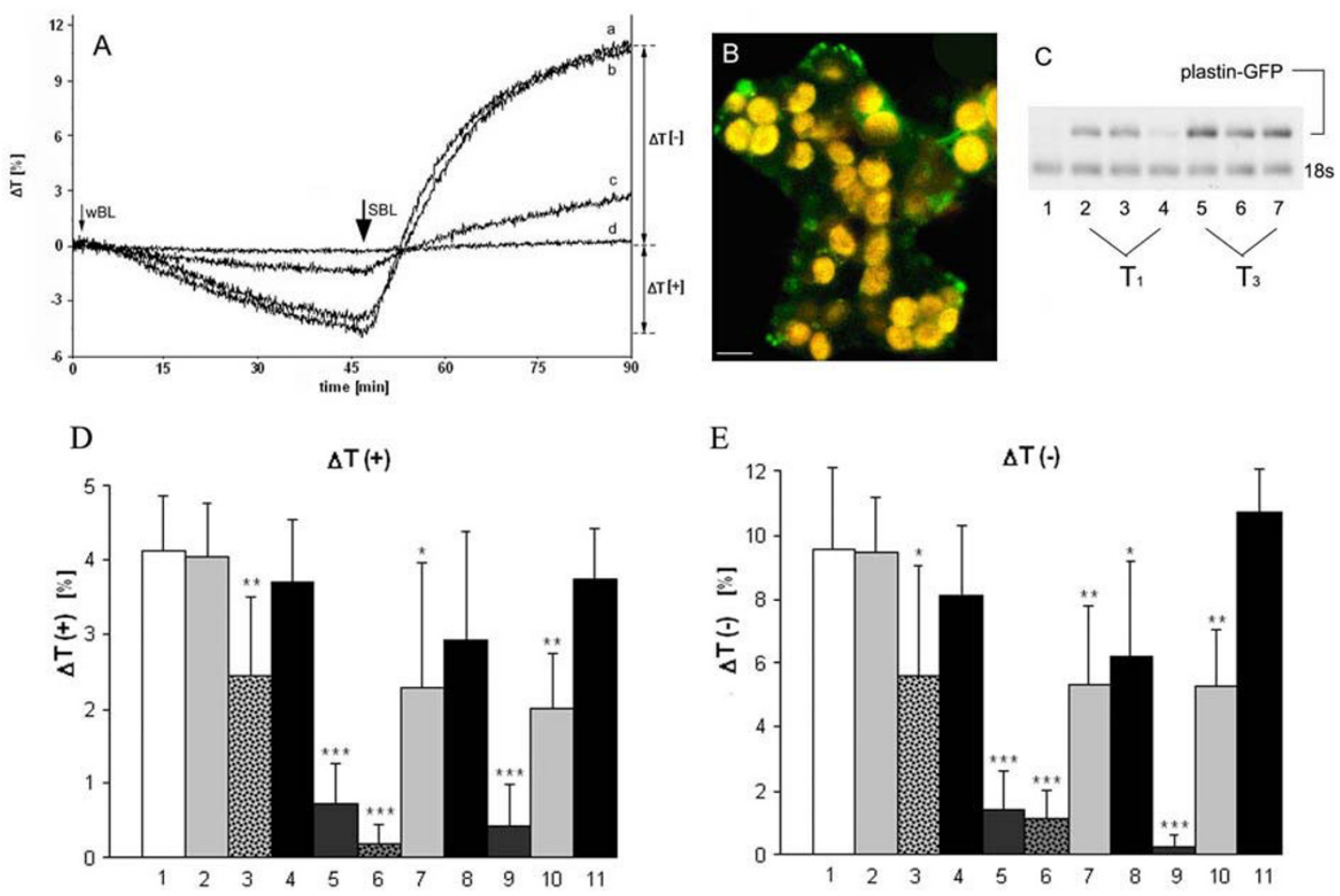

E
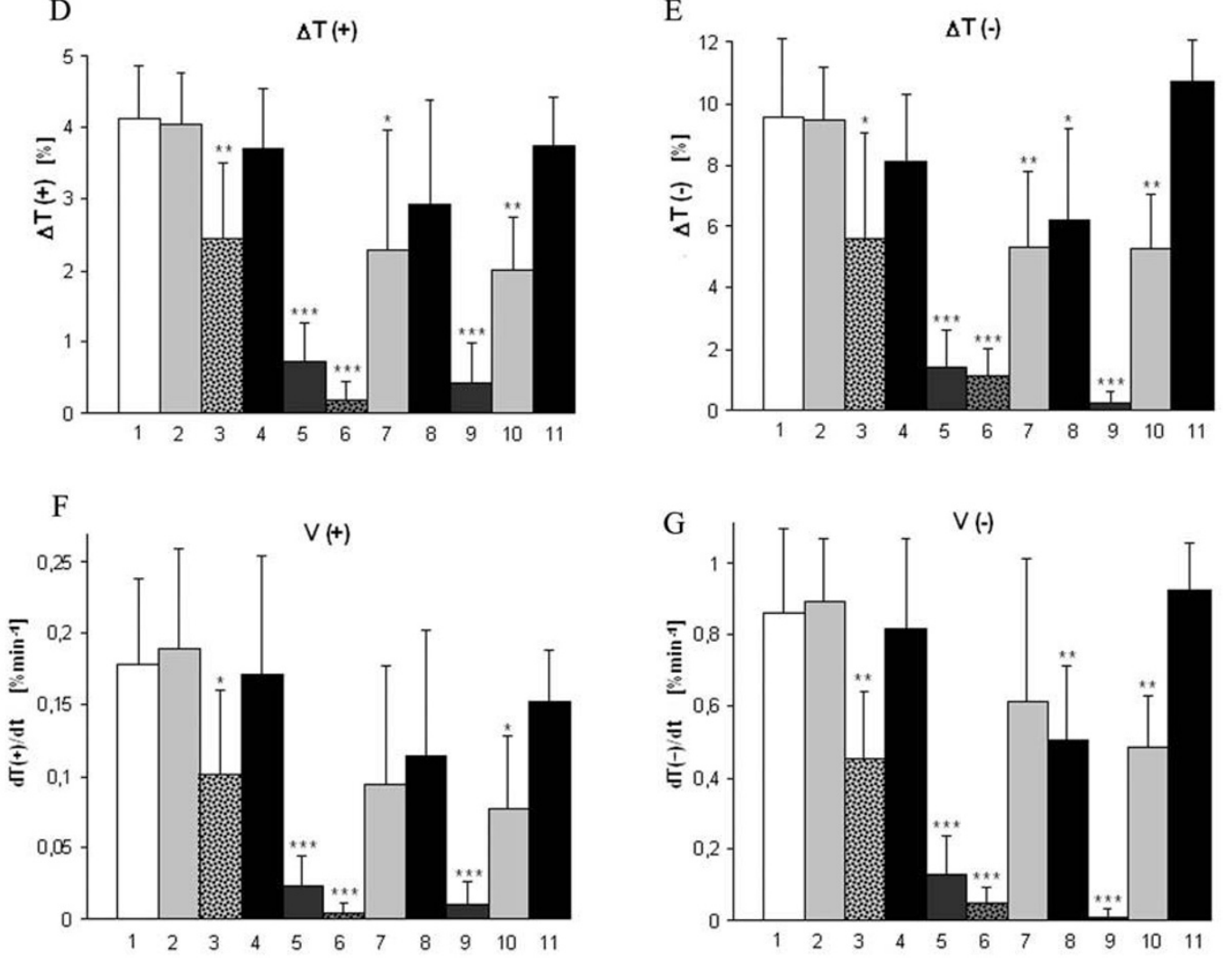

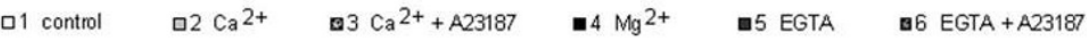

$$
\begin{aligned}
& \square 7 \mathrm{Ca}^{2+} \text { after EGTA } \quad 8 \mathrm{Mg}^{2+} \text { after EGTA } \quad 9 \text { TFP } \square 10 \mathrm{Ca}^{2+} \text { after TFP }=11 \mathrm{Mg}^{2+} \text { after TFP }
\end{aligned}
$$

Figure I

(A) Chloroplast responses to blue light in wild-type and transgenic N. tabacum cv. Samsun expressing plastinGFP. The curves show changes in transmission of red measuring light $(\Delta T)$ through dark-adapted leaves exposed to continuous weak blue light ( $\mathrm{WBL}, 0.4 \mathrm{Wm}^{-2}, 45 \mathrm{~min}$ ) and strong blue light (SBL, $\left.10 \mathrm{Wm}^{-2}, 45 \mathrm{~min}\right) . \Delta \mathrm{T}[+]$ and $\Delta \mathrm{T}$ [-] denote amplitudes of accumulation and avoidance responses respectively. Representative responses for about seventy tests carried out with three-month-old wild-type (a) and transgenic (b) tobacco plants. Curves c and d are representative of two-month (c) and onemonth-old (d) plants. (B) Actin organization in immature mesophyll of transgenic (one-month-old) plants grown

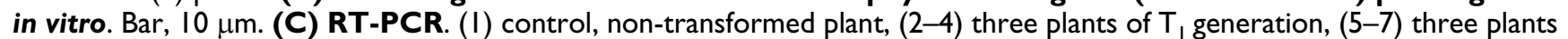
of $T_{3}$ generation. (D - G) Parameters of blue light-controlled chloroplast responses in mature leaves of transgenic N. tabacum. (D, E) Amplitudes: $\Delta \mathrm{T}(+)$ of weak (wBL, $\left.0.4 \mathrm{Wm}^{-2}\right)$, and $\Delta \mathrm{T}(-)$ of strong $\left(\mathrm{SBL}, 10 \mathrm{Wm}^{-2}\right) \mathrm{blue} \mathrm{light}$ responses. $(F, G)$ Velocities: $V(+)$ of wBL, and $V(-)$ of SBL responses. Averages of 7-I4 measurements. Error bars represent $\mathrm{SD}$. Asterisks denote the significance of differences ( $\mathrm{p}$-value calculated with the unpaired t-test, $* \mathrm{p}=0,05-0,00 \mathrm{I} ; * * \mathrm{p}=0,00 \mathrm{I}-$ 0,0001 ; *** $p<0,000$ I). 


\section{General outline of experiments}

Samples from one leaf, subjected to identical treatment, were concomitantly used for measuring the movement activity of chloroplasts and for testing AC by confocal microscopy. Chloroplast movements were activated with blue light. To seek BL-specific effects on the organization of $\mathrm{AC}$, the samples tested microscopically were irradiated with blue or red light. Red light, inactive in chloroplast redistribution, was used as control. The effects of compounds disturbing calcium homeostasis (EGTA alone or supported by calcium ionophore, trifluoperazine) were compared in the above two experimental settings, and then $\mathrm{Ca}^{2+}$ ions were added to counteract the antagonists. Because earlier investigations showed that $\mathrm{Mg}^{2+}$ could eliminate the inhibitory effects of EGTA on chloroplast redistribution [23], all experiments were also repeated with $\mathrm{Ca}^{2+}$ substituted by $\mathrm{Mg}^{2+}$. Visualization of changes in the cytoskeleton was supported by quantitative image analysis. Additionally, a potential interaction between $\mathrm{Ca}^{2+}$ and phosphoinositide-mediated signal transduction pathways was tested by compensating wortmannin inhibitory effects on chloroplast responses with addition of both divalent cations.

\section{Actin organization in continuous light}

In dark-adapted cells distinctly outlined actin bundles formed a branched network (Fig. 2A, B). The chloroplasts were associated with basket-shaped structures consisting of thin AFs (arrows). These baskets were tightly bound together around adjacent chloroplasts and attached to cortical actin bundles. Circular structures of various sizes were sporadically seen in the cytoplasm (Fig. 2A, arrowheads). Numerous small loops were present, predominantly on the surface of chloroplasts (Fig. 2B, arrowheads). Most of them contained mitochondria (Fig. 2b arrowhead).

The organization of AC was modified after irradiation with wBL. This light induced an accumulation response of chloroplasts, shown in Fig. 1A and 1D as decrease of light transmission $\Delta \mathrm{T}(+)$ through the leaf. The AFs reorganized without losing their clear-cut appearance (Fig. 2C). The quantitative analysis showed a distinct narrowing of actin bundles in weak light (Fig. 3). Single chloroplasts were wrapped in discrete bundles finer than those present in the dark (Fig. 2c; for quantitative evaluation see Additional file 1, Ctrl: Energy of actin distribution pattern in Factin baskets surrounding chloroplasts). The general distribution of mitochondria with respect to chloroplasts did not change as compared with the dark-adapted material (not shown).

After the tissue was exposed to SBL the image of almost all cell actin became diffuse with occasional single wide strands to which chloroplasts were attached (Fig. 2D, arrows). The chloroplasts took on the profile position characteristic of the avoidance response $(\Delta \mathrm{T}[-]$ in Fig. $1 \mathrm{~A}$ and $1 \mathrm{E}$, Additional file 2). The baskets on chloroplast surfaces became diffuse but the small loops containing mitochondria were still conspicuous (Fig. 2d). The connection between the baskets and the actin network appeared looser than in the dark-adapted or wBL-treated cells. The F-actin image started to get diffuse as early as several min after SBL irradiation even if the tissue had been pre-irradiated with wBL. The wide strands were seen reorganizing upon irradiation with strong light (Additional files 3, 4 and 5). The "diffusion effect" was reversible and the reconstruction of a distinct, branched actin network took place in the SBL-irradiated samples subsequently treated with continuous $\mathrm{wBL}$ (Additional file 6). This reconstruction was observed no sooner than 60 min after the onset of weak light exposure.

The effects of blue light were compared with those produced by red light (RL). Almost the same images of the actin network were obtained after exposure to BL or RL with equivalent quantum fluxes (Fig. 2E, F). Strong light produced indistinguishable images of widened F-actin bundles and foamy chloroplast baskets irrespective of wavelength. Hence, no blue-specific differences could be detected in the actin structure. The quantitative results in Fig. 3 (Ctrl) and Additional file 1 (Ctrl) support this observation.

\section{Effects of $\mathrm{Ca}^{2+}, \mathrm{Ca}^{2++}$ ionophore $\mathrm{A23} / 87$ and $\mathrm{Mg}^{2+}$}

The effect of extracellular calcium on the structure of the actin cytoskeleton and on chloroplast movements was studied using a $5 \mathrm{mM}$ solution of calcium nitrate. The image of AC became more distinct after the addition of $\mathrm{Ca}^{2+}$. The effect was visible in the dark-adapted mesophyll cells and even stronger after wBL or wRL irradiation (Figs $3,4 \mathrm{~A}$ and $4 \mathrm{C}$ ). Photometric transmission changes reflecting the chloroplast responses to $\mathrm{BL}$ were about the same as with the control (Fig. 1D-G, bar 2). Exposure of the of $\mathrm{Ca}^{2+}$-treated tissue to SBL and SRL produced similar wide bands of AFs with side adhering chloroplasts. All formations observed in these images looked very similar to those from SL-irradiated controls (Fig. 3, Additional file 7).

The concomitant application of $\mathrm{Ca}^{2+}$ ionophore A23187 worsened the cytoskeleton image typical of the ambient $\mathrm{Ca}^{2+}$-solution, particularly after exposure to either wBL or wRL (data not shown). The addition of ionophore A23187 to the calcium nitrate solution reduced amplitudes by about $40 \%$ and velocities by about $50 \%$ in both chloroplast responses (Fig. 1D, G, bar 3).

As shown in Fig. 4 the actin cytoskeleton at higher extracellular $\mathrm{Mg}^{2+}$ concentration looked similar to that at the 

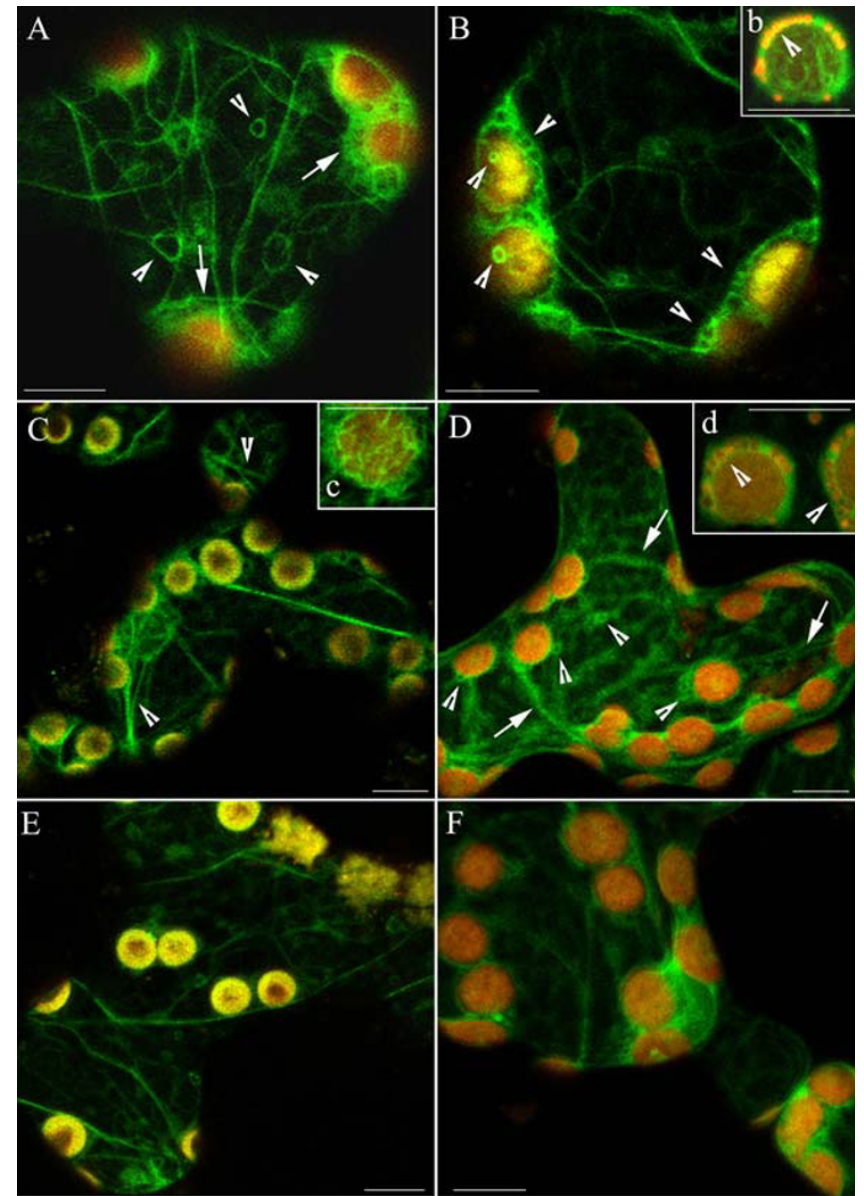

Figure 2

The actin cytoskeleton in 3-month-old tobacco mesophyll cells as visualized by plastin-GFP (green fluorescence). (A, B) Network of actin in dark-adapted cells. "Baskets" around chloroplasts marked with arrows and circular structures marked with arrowheads; yellow-red colour comes from autofluorescence of chloroplasts. (C) Reorganization of F-actin after I h of exposure to continuous WBL $\left(0.4 \mathrm{Wm}^{-2}\right)$. Strands which spread across the cortical cytoplasm, frequently split into thinner filaments (marked with arrowheads). (c) Actin filaments forming baskets are better resolved after wBL. (D) Wide bands of F-actin (arrows) have a loose contact with chloroplasts after exposure to SBL ( 10 $\left.\mathrm{Wm}^{-2}, 20 \mathrm{~min}\right)$. (E) Effect of continuous wRL $\left(0.24 \mathrm{Wm}^{-2}\right.$, I h) or (F) to SRL $\left(6.7 \mathrm{Wm}^{-2}, 20 \mathrm{~min}\right)$ on the actin cytoskeleton. Scale bars, $10 \mu \mathrm{m}$. The cytoskeleton forms numerous small loops ( $B$ and $D$, arrowheads), most of them containing mitochondria. Insets $b$ and $d$ show magnified chloroplasts with mitochondria visible (orange/red colour) in the $A C$ loops after staining with TMRE.

presence of $\mathrm{Ca}^{2+}$ both in dark-adapted and wL-irradiated tissue. The tendency to widening was occasionally visible in strong light (see Additional file 6) but the quantitative analysis showed that $\mathrm{Mg}^{2+}$ ions subdue the strong light effect (compare striped bars in Fig. 3). The parameters of chloroplast responses were somewhat lower with $\mathrm{Mg}^{2+}$ than with either $\mathrm{Ca}^{2+}$ or the control, however, the difference was statistically insignificant (Fig. 1D-G, compare bars 4 and 2).

\section{Effects of EGTA and EGTA + calcium ionophore A23 187}

Potent inhibition of chloroplast responses concomitant with dramatic changes in AC organization were observed in tissue incubated with $1 \mathrm{mM}$ EGTA for $30 \mathrm{~min}$ to $1 \mathrm{~h}$ in the dark (Fig. 1D-G, bar 5; Fig. 5A). EGTA caused the formation of a characteristic spotted pattern all over the cell. At the same time, a dense network of fine AFs formed at the chloroplast surfaces (Fig. 5a). Loops of various sizes with a fluorescent-green tint inside were visible in some cells (Fig. 5A, arrowheads). Numerous chloroplasts were grouped into tight clusters (asterisk). After exposure to weak light, the spotted pattern persisted but the chloroplast baskets became clearly visible: very fine filaments appeared on the surfaces of chloroplasts (Fig. 5B, arrowheads). The energies of F-actin patterns corresponding to chloroplast baskets were 33.7 for dark-adapted vs 37.1 for wB (99th percentile, arbitrary units). The increase in energy signifies growing inhomogeneity of AC i.e. the emergence of distinct AFs. This difference was detectable at all percentiles (not shown). The structure characteristic of treatment with EGTA disappeared in strong light. Simultaneously, filamentous actin reappeared in the cells in the form of a few widened strands (Additional file $7 \mathrm{C}$ and 7D).

When EGTA was applied together with the calcium ionophore, both accumulation and avoidance responses were almost eradicated (Fig. 1D-G, bar 6). Strong inhibition of the chloroplast movements was observed as early as 30 min after the beginning of incubation. Along with the arrest of movement, the addition of the calcium ionophore accelerated and intensified changes in the $\mathrm{AC}$ organization produced by EGTA (cf. Fig. 5 and Additional file 8).

\section{Reversion of EGTA effects by $\mathrm{Ca}^{2+}$ and $\mathbf{M g}^{2+}$}

Calcium and magnesium ions reversed the damage caused by EGTA in the dark-adapted cells when used directly after the chelating agent (Fig. 5C, E). Irradiation with $\mathrm{wL}$ helped the reconstruction of the filamentous structure of actin. Sharper AFs were restored in the presence of $\mathrm{Mg}^{2+}$ than in the presence of $\mathrm{Ca}^{2+}$ (Fig. 5D, F). Both ions caused the chloroplasts to separate from the EGTA-induced clusters (Fig. 5C-F). While the structure of the actin network was considerably improved by $\mathrm{Ca}^{2+}$, the chloroplast responses were reactivated only by $50 \%$ (Fig. 1D-G, bar 7). As with its effect on the cytoskeleton, $\mathrm{Mg}^{2+}$ restored both chloroplast responses to blue light somewhat better than $\mathrm{Ca}^{2+}$ (Fig. 1D-G, bar 8, contrast with 7). 


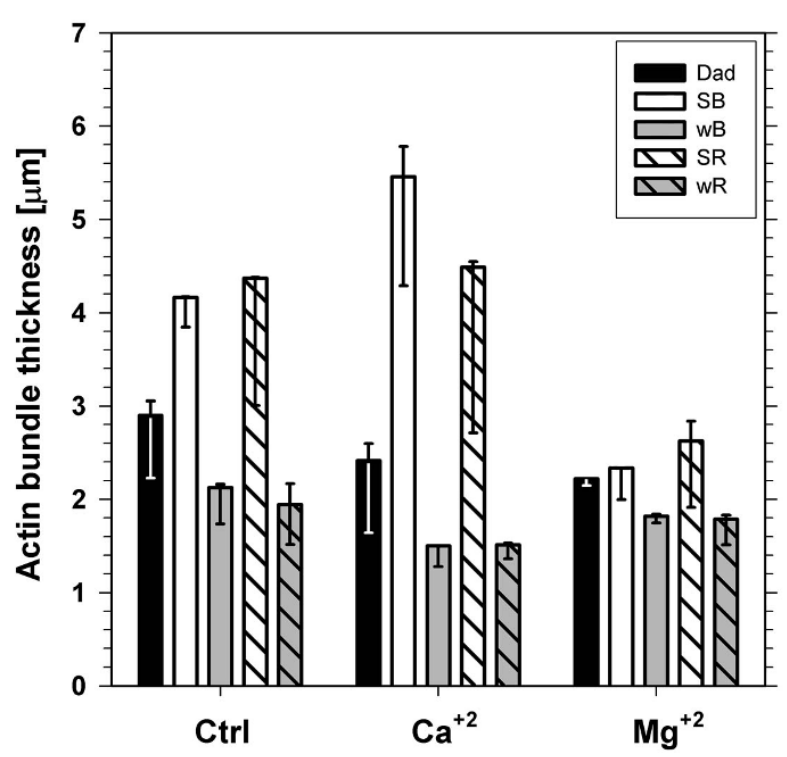

Figure 3

Thickness of actin bundles in control (Ctrl) and in the presence of calcium $\left(\mathrm{Ca}^{+2}\right)$ and magnesium $\left(\mathrm{Mg}^{+2}\right)$

ions. The bundle thickness was measured in cells adapted to darkness (Dad, black bars) and in cells illuminated with strong (SB) or weak (wB) blue light (empty and gray bars, respectively) and with strong (SR) or weak (WR) red light (backslashed and backslashed gray bars, respectively). The thickness was calculated in micrometers $(\mu \mathrm{m})$ as 99 th percentile of the data corresponding to averages of optical sections, whereas error bars represent $95 \%$ confidence intervals.

\section{Effects of trifluoperazine and their reversion by $\mathrm{Ca}^{2+}$ and $\mathrm{Mg}^{2+}$}

Trifluoperazine (TFP, $20 \mu \mathrm{M}$ ), a blocker of calmodulin caused destabilization of the AC as early as $15 \mathrm{~min}$ after application. Chloroplast clusters formed in most cells as with EGTA (Fig. 6A, compare with Fig. 5A, asterisks). ACassociated fluorescence disappeared after $1 \mathrm{~h}$ of incubation in the dark (Fig. 6a). Early exposure to light, especially $\mathrm{wBL}$, brought about a reconstruction of actin bundles and chloroplast separation (Fig. 6B). In spite of AC recovery, the chloroplasts did not respond to light in the TFP-treated tissue. The first disturbances were detected after $15 \mathrm{~min}$, and $15 \mathrm{~min}$ later the avoidance response was practically extinguished. Both responses were completely inhibited after 45 min treatment with TFP (Fig. 1D-G, bar 9).

The effect of TFP was reversed by calcium and magnesium in the dark (Fig. 6C, D). Both ions caused the chloroplasts to separate from the TFP-produced clusters. Even though both ions prompted the recovery of chloroplast movements, magnesium was notably twice as effective as cal- cium in the reactivation (Fig. 1D-G, bar 11, contrast with $10)$.

Irrespective of the ionic/pharmacological treatment, the effects of BL and RL on cortical AC were comparable. Irradiations with equivalent quantum fluxes of SB and SR and/or wB and wR resulted in formation of F-actin patterns of similar energies, respectively. No blue-specific differences could be detected (see Additional file 1).

\section{Effects of wortmannin}

Wortmannin (WM), an inhibitor of phosphoinositide-3kinase, had a dramatic effect on chloroplast movements at a concentration of $10 \mu \mathrm{M}$ (Fig. 7A). The accumulation response was eliminated and the avoidance response was reduced by half after $1.5 \mathrm{~h}$ exposure. Again, $\mathrm{Ca}^{2+}$ and $\mathrm{Mg}^{2+}$ negated the inhibition, with full recovery of the avoidance response obtained with both investigated ions. The influence of WM was stronger on velocities than on amplitudes, but they were similarly reactivated by $\mathrm{Ca}^{2+}$ and $\mathrm{Mg}^{2+}$ (data not shown). In this case, however, the inhibitory effect on the movement was not reflected in the shape of cellular actin: AC remained completely unaffected by WM (Fig. 7B). Only when the concentration was increased to $50 \mu \mathrm{M}$ did some perturbations in the continuity of actin bundles become perceptible (Fig. 7C). This higher concentration of WM abolished both chloroplast responses to light.

\section{Discussion}

The widening of actin strands upon SL-irradiation can be interpreted as a relaxation of the structure of actin bundles. It might be a result of some yet undefined interaction between filaments (or between actin and ABPs) in SL, leading to the formation of looser bundles. The differences between the organization of cytoskeleton in wL and SL may have consequences for the manner in which actin anchors the chloroplasts in a cell under different light conditions. The tendency of chloroplasts to be displaced during centrifugation was investigated in Lemna trisulca, a model species used in studies on blue-activated chloroplast movements in higher plants. The ability of chloroplasts to resist centrifugal force depended on light pretreatment. Whereas $\mathrm{wL}$ anchored chloroplasts in the cells, SL loosened the binding and made them easier displaceable by centrifugal forces [29]. The relaxation reported currently might be the basis of the mentioned SL effect.

Circular forms of AFs sometimes occurred in dark-adapted or wBL-treated tobacco cells. Similar forms had previously been observed in fixed Adiantum protonemal cells and assigned a role in chloroplast anchoring [30]. However, in Adiantum the circles had a much bigger diameter and were found only in irradiated tissue. 


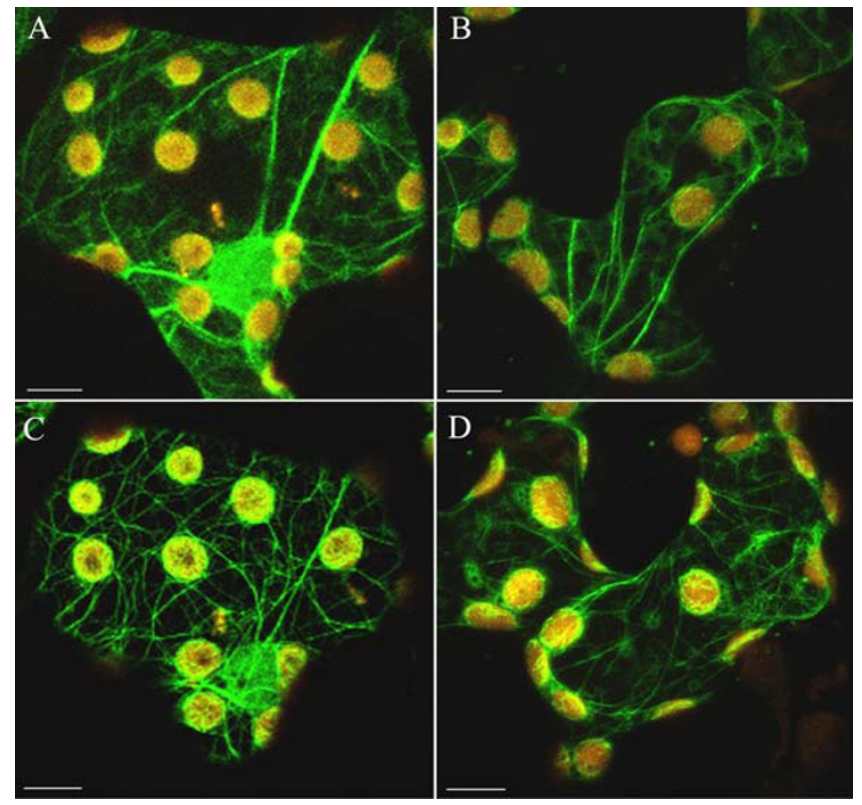

Figure 4

Influence of calcium and magnesium ions on the actin network in dark-adapted and weak light-irradiated cells. Samples were incubated for $2 \mathrm{~h}$ with $5 \mathrm{mM} \mathrm{Ca}{ }^{2+}$ $(A, C)$ or $5 \mathrm{mM} \mathrm{Mg}^{2+}(B, D)$. Actin cytoskeleton in the darkadapted cells $(A, B)$ and after irradiation with continuous weak red light for I h (C, D). Scale bars, $10 \mu \mathrm{m}$.

The differences observed in tobacco are in contrast to the results obtained with fixed tissue of $A$. thaliana, where no structural dissimilarities of actin were detected between leaves treated with wBL or SBL [27]. The different susceptibility of actin to light in Arabidopsis as compared to Nicotiana might be attributed to a non-identical organization of the filament bundles in these species. This could account for three unsuccessful attempts to transform Arabidopsis leaves with the plastin-GFP construct used in this study whereas siliques and sepals showed an effective transformation (unpublished data from our laboratory). On the other hand, the discrepancy between the actin images obtained for SBL-irradiated tobacco and Arabidopsis could be a consequence of the fixation procedure used for the latter species.

Seeking BL-specific actin reorganization we compared the effects of blue and red light on the actin cytoskeleton. As with fixed cells, RL and BL effects were similar, even though RL induces no directional chloroplast movement in tobacco [31]. This lack of difference confirms our previous conclusion that the directionality of chloroplast responses is not based on BL-specific changes of F-actin, and that other factor(s) must determine the direction of chloroplast movement.
The presence of the dense cortical F-actin network associated with chloroplast baskets was demonstrated in living tobacco cells as in other reports dealing with fixed actin in Arabidopsis $[13,27]$. The structure of the actin baskets and their interactions with the cortical AC seem to be of key importance for chloroplast positioning in higher land plants. Even though irradiation with wL and/or SL changed the structure of actin baskets in living cells, they were always tightly associated with chloroplast surfaces.

The significant improvement of the cytoskeleton image by $\mathrm{Ca}^{2+}$ and $\mathrm{Mg}^{2+}$ may be due to the binding of these ions to either AFs or plastin. Contrary to our results, external calcium and magnesium had no visible effect on the phalloidin-labelled AC organization in tobacco BY-2 protoplasts [32]. In poppy pollen tubes, F-actin was fragmented into punctate foci at increasing concentration of cytosolic free $\mathrm{Ca}^{2+}[33]$.

The damage to AC made by external EGTA depleting the cytosolic $\mathrm{Ca}^{2+}$ confirms the obvious fact that calcium is important for the maintenance of microfilament integrity. The formation of the well structured filamentous actin on the surfaces of chloroplasts (Fig. 5B) may be due to $\mathrm{Ca}^{2+}$ extraction from these organelles during $w B L$ irradiation. Blue light of $1 \mu \mathrm{mol} \mathrm{m}{ }^{-2} \mathrm{~s}^{-1}$ produced a transient increase in cytosolic $\mathrm{Ca}^{2+}$ in A.thaliana leaves, which originated partly from internal calcium stores [5]. Our results can be interpreted in terms of BL causing an efflux of calcium from chloroplasts, and its continuous chelating by extracellular EGTA. This combined action of light and EGTA might have induced the transient formation of fine actin baskets on chloroplast surfaces, whereas EGTA prevented a stable restoration of actin cytoskeleton in the cells.

Along with the destruction of the actin network, EGTA strongly inhibited chloroplast responses in tobacco, similar to the effects previously reported for ferns and water angiosperms $[24,25]$. Combined treatment with EGTA and A23187 merely accelerated and intensified the effects of EGTA. The ionophore facilitated the efflux of calcium from the cells.

Trifluoperazine, a blocker of calmodulin completely inhibited the chloroplast movements in tobacco, similar to the inhibition previously reported for Lemna [24]. TFP also caused the disappearance of almost all AC-related fluorescence after $1 \mathrm{~h}$ dark-incubation. Light effected a partial reconstruction of AFs in the presence of TFP, most probably due to an increase in the cytosolic $\mathrm{Ca}^{2+}$ concentration. Remarkably, chloroplast responses were not restored. Even though $\mathrm{Ca}^{2+}$ presumably released by $\mathrm{BL}$ efficiently rebuilt the TFP-destroyed AFs, it was not capable of reactivating chloroplast responses. This may suggest 
that calmodulin per se is involved in transmitting the directional signal.

Both $\mathrm{Ca}^{2+}$ and $\mathrm{Mg}^{2+}$ effectively restored the EGTA- and TFP-damaged actin cytoskeleton. The differences between the actin bundles restored by these ions might be attributed to differences in de novo actin polymerization. In a study on rabbit skeletal muscle, $\mathrm{Mg}^{2+}$ played a stronger role in the mechanism of actin polymerization than $\mathrm{Ca}^{2+}$ due to faster nucleation of Mg-ATP-actin than Ca-ATPactin [34]. Indeed, Mg-ATP-actin nucleates three orders of magnitude faster than Ca-ATP-actin $[35,36]$. Besides, actin containing tightly-bound $\mathrm{Mg}^{2+}$ differs structurally and functionally from actin containing tightly-bound $\mathrm{Ca}^{2+}[37,38]$.

Both ions were shown to restore not only the actin cytoskeleton, but also chloroplast responses in EGTA/TFP treated tobacco cells. $\mathrm{Ca}^{2+}$ has previously been reported to restore the EGTA-inhibited chloroplast photo-orientation in Adiantum protonemal cells [39]. $\mathrm{Mg}^{2+}$ has been shown to counteract the inhibitory effect of EGTA in Lemna when applied together with the chelator [24]. It was hypothesized that $\mathrm{Mg}^{2+}$ blocked the calcium channels through which $\mathrm{Ca}^{2+}$ was removed from the cell by the external EGTA. Extracellular magnesium has indeed been shown to significantly modifiy the transport of all major ions, $\mathrm{H}^{+}$, $\mathrm{Ca}^{2+}$, and $\mathrm{K}^{+}$in bean mesophyll cells [40].

Remarkably, $\mathrm{Mg}^{2+}$ was twice as effective in restoring chloroplast photo-responses than $\mathrm{Ca}^{2+}$ in samples pre-treated with TFP. How was $\mathrm{Mg}^{2+}$ able to fully recover the directional chloroplast movement? Could it act indirectly, bypassing calmodulin or triggering other pathways that substitiute for calmodulin activity? It is difficult to answer these questions because magnesium homeostasis is still poorly understood [41]. The molecular details of $\mathrm{Mg}^{2+}$ transport between cellular compartments in plants are still far from clear $[42,43]$. $\mathrm{Mg}^{2+}$ ions are stored mainly in vacuoles. A large part of the cytoplasmic magnesium is complexed by ATP. The concentration of free $\mathrm{Mg}^{2+}$ in the cytosol must therefore be strictly regulated, which is a precondition for playing a role in signal transduction [42]. In animal systems, magnesium has been postulated as acting as an intracellular messenger [41]. Could it play such a role also in plant cells?

The strong inhibition of chloroplast movement by wortmannin shows that the model assigning phosphoinositide kinases a key role in the transduction of the orienting BL signals in Lemna [26] may be valid also for higher land plants. On the other hand, the model needs further refinement. Firstly, WM at a concentration of 10 $\mu \mathrm{M}$, which is strongly inhibitory for both chloroplast responses, had no effect on tobacco AC. Thus, the BL sig- nals are not directed to actin, which is consistent with our former conclusion $[12,27]$. Small disturbances in the network were perceptible only at $50 \mu \mathrm{M}$, above the range of concentrations commonly used in plants $[26,44]$. Secondly, the striking recovery of WM-inhibited movements obtained with $\mathrm{Ca}^{2+}$ shows that this ion is not only needed for controlling the motor apparatus (myosin) but also that it transmits the signal downstream of the phosphoinositide kinases. Our results suggest therefore further complications in the model of signal transduction.

All investigated remedial activities of extracellular $\mathrm{Ca}^{2+}$ could be also mimicked by $\mathrm{Mg}^{2+}$, in most cases even more efficiently. Thus, our results point to the possibility that $\mathrm{Mg}^{2+}$ is a regulatory molecule cooperating with $\mathrm{Ca}^{2+}$ in the signaling pathway of BL-induced tobacco chloroplast movements. It has to be stressed that extracellular $\mathrm{Ca}^{2+}$ / $\mathrm{Mg}^{2+}$ reactivated the directional movements even though applied non-directionally. Thus, an asymmetric, polar distribution of some yet undisclosed cellular elements with which this(these) ion(s) interact seems to be required to define the direction of chloroplast movements in higher plants.

\section{Conclusion}

The actin cytoskeleton in the mesophyll of tobacco is sensitive to weak and strong light irrespective of its spectral region (blue or red). Thus, the directionality of chloroplast responses is not based on specific blue light-induced changes of F-actin but on other, yet unidentified factor(s).

The structure of the actin baskets surrounding chloroplasts, and their interactions with the cortical actin cytoskeleton appear to be crucial for chloroplast positioning in higher land plants.

The striking recovery of wortmannin-inhibited movements obtained with $\mathrm{Ca}^{2+}$ shows that these ions play at least two roles in the mechanism of the movements: they control the motor apparatus and transmit the light-generated signal downstream of the phosphoinositide kinases.

Our results show for the first time that $\mathrm{Mg}^{2+}$ is a regulatory molecule cooperating with $\mathrm{Ca}^{2+}$ both in the maintenance of the actin network integrity and in the signaling pathway of chloroplast movements in tobacco.

\section{Methods}

\section{Plant growth conditions}

Nicotiana tabacum plants (ecotype Samsun) used for experiments were grown on MS medium supplied with Gamborg vitamins, 3\% (w/v) sucrose and solidified with $0.8 \%(\mathrm{w} / \mathrm{v})$ agar. The axenic cultures were kept in a growth chamber (Sanyo MLR-350, Japan) equipped with fluorescent tubes (Sanyo FL 40SS.W/37 and OSRAM L 36W/77 

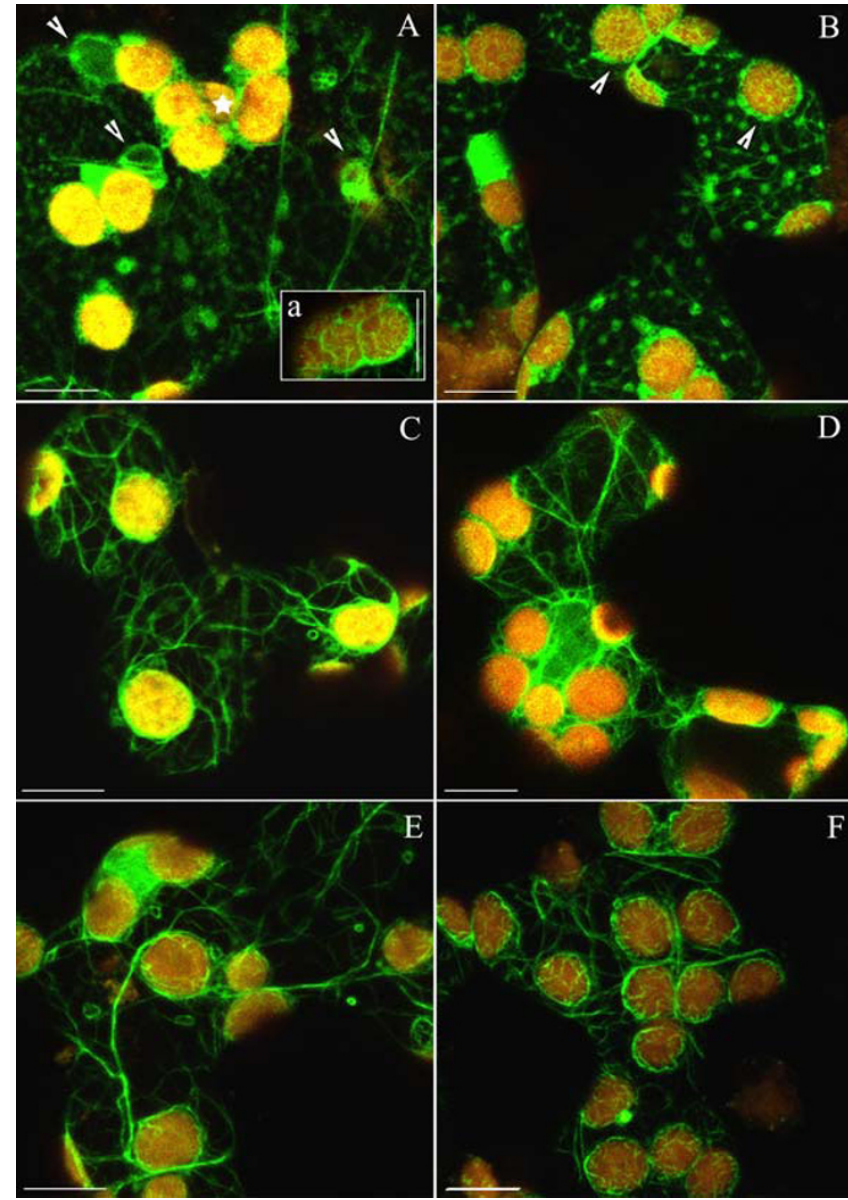

Figure 5

Disintegration of F-actin in EGTA and restoration of actin network prompted by calcium or magnesium ions. (A) Formation of actin foci in response to 0,5-I h incubation with I mM EGTA in dark-adapted cells. Fluorescent spots and loops of various sizes (arrowheads) are visible throughout the cytoplasm. Chloroplasts are arranged into tight clusters (asterisk). Thin filaments are present on chloroplast surfaces (a). (B) Actin foci persist after wBL irradiation. Distinct baskets around chloroplasts (arrowheads) became more visible after exposure to weak light. Effect of $5 \mathrm{mM}$ $\mathrm{Ca}^{2+}(\mathrm{C}, \mathrm{D})$ or $5 \mathrm{mMMg}{ }^{2+}(\mathrm{E}, \mathrm{F})$, each applied for $2 \mathrm{~h}$ on actin organization in EGTA pre-treated cells. In both cases, F-actin network recovered in dark-adapted cells (C, E) and after additional exposure to continuous wBL for I $h(D, F)$. Scale bars, $10 \mu \mathrm{m}$.

Fluora, Germany). The fluence rate of the fluorescent light was 60 to $100 \mu \mathrm{mol} \mathrm{m} \mathrm{m}^{-2} \mathrm{~s}^{-1}$. The photoperiod was $12 / 12 \mathrm{~h}$ and the temperature was $23^{\circ} \mathrm{C}$.

\section{Constructs, plant transformation and bacterial growth conditions}

Tobacco was stably transformed using the Agrobacterium tumefaciens strain LBA 4404 containing the binary plas- mid pBI 121 (for more details see [27]). The plasmid carried a gene coding for a fusion protein consisting of truncated human plastin and smGFP (plastin-GFP) under the control of the cauliflower mosaic virus $35 \mathrm{~S}$ promoter. The Agrobacterium was cultured for two days in the dark at $28^{\circ} \mathrm{C}$ in $\mathrm{LB}$, a liquid medium supplemented with $20 \mathrm{mg} \mathrm{l}^{-}$ ${ }^{1}$ rifampicin and $100 \mathrm{mg} \mathrm{l}^{-1}$ streptomycin. Bacteria $\left(\mathrm{OD}_{600}\right.$ 1.6) were resuspended in $5 \mathrm{ml}$ of liquid medium containing MS salts supplemented with Nitsch vitamins, $0.2 \%$ $(\mathrm{w} / \mathrm{v})$ glucose, $0.004 \%(\mathrm{w} / \mathrm{v})$ adenine, $1.0 \mathrm{mg} \mathrm{l}^{-1} \mathrm{BAP}$ and $0.1 \mathrm{mg} \mathrm{l}^{-1} \mathrm{NAA}$. Tobacco leaf discs were inoculated with bacterial suspension and co-cultured for two days in the dark at $28^{\circ} \mathrm{C}$ in the same medium solidified with agar. The regeneration/selection was carried out on a medium containing MS salts with Gamborg vitamins, 3\% (w/v) sucrose, $40 \mathrm{mg} \mathrm{l}^{-1}$ adenine, $1 \mathrm{mg} \mathrm{l}^{-1} \mathrm{BAP}, 0.1 \mathrm{mg} \mathrm{l}^{-1} \mathrm{NAA}$, carbenicillin to kill the bacteria and kanamycin to inhibit growth of non-transformed plant cells. The carbenicillin content was gradually reduced from 500 to $200 \mathrm{mg} \mathrm{l}^{-1}$, while the kanamycin concentration was kept at $50 \mathrm{mg} \mathrm{l}^{-1}$. The transformed cells grew into callus and differentiated into shoots via organogenesis. Two generations of transgenic plants were tested for the presence of plastin-GFP under a confocal microscope. One plant exhibiting the most distinct and uniform expression in the mesophyll cells was reproduced vegetatively and cultured under axenic conditions. Three-month-old transgenic leaves of these plants were used for experiments. The vegetative culture was continued for 15 months with plants transferred to fresh medium every 3 months.

Control and transgenic plants obtained from first and third generation seeds were used for RT-PCR. Total RNA obtained with RNeasy Plant Mini Kit (Qiagen GmbH, Germany) and decontaminated from DNA with DNAfreeTM Kit (Ambion Europe Ltd UK) was used for cDNA synthesis with random hexamer primers (RevertAidTM First Strand cDNA Synthesis Kit; Fermentas UAB, Lithuania). The semi-quantitative RT-PCR was carried out after normalization with QuantumRNATM 18S RNA (Ambion Europe Ltd UK, 3:7 primer:competimer ratio), an internal control. Primers were designed using Biology WorkBench 3.2 (plastin-GFP left primer 5'-CTGACATTGAATTAAGCAGGAATG-3' and right primer 5'-AAGCATTGAACACCATAAGTGAAA-3').

\section{Treating solutions}

All solutions were buffered with $10 \mathrm{mM}$ PIPES, piperazine-1,4-bis(2-ethanesulphonic acid). The following treating solutions were used: $5 \mathrm{mM} \mathrm{Ca}\left(\mathrm{NO}_{3}\right)_{2} ; 5 \mathrm{mM}$ $\mathrm{Mg}\left(\mathrm{NO}_{3}\right)_{2} ; 10 \mu \mathrm{M}$ calcium ionophore A23187 (4-bromocalcimycin A23187); calcium-free solution: $1 \mathrm{mM}$ $\mathrm{EGTA}+1.5 \mathrm{mM} \mathrm{KH}_{2} \mathrm{PO}_{4}+5 \mathrm{mM} \mathrm{KNO}_{3} ; 20 \mu \mathrm{M}$ trifluoroperazine; $10 \mu \mathrm{M}$ and $50 \mu \mathrm{M}$ wortmannin. The ionophore and wortmannin were initially dissolved in DMSO and 


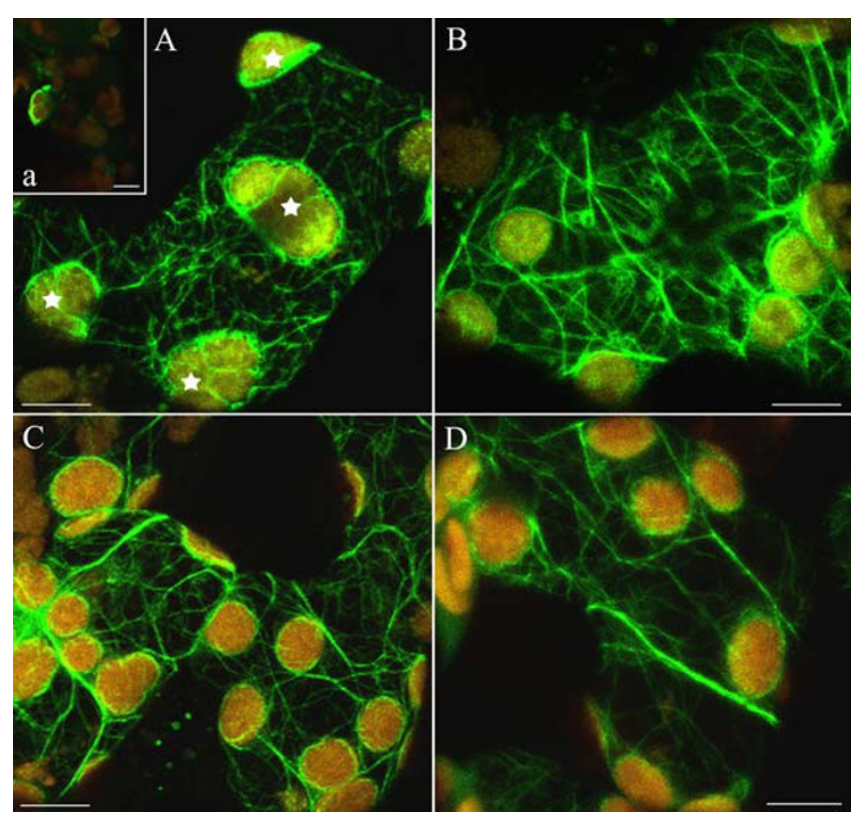

Figure 6

Disintegration of actin bundles by trifluoperazine and its reversal by $\mathbf{C a}^{2+}$ and $\mathbf{M g}^{2+}$.(A) Images of disordered $\mathrm{F}$ actin after treatment with $20 \mu \mathrm{M}$ TFP for $30 \mathrm{~min}$; chloroplast clusters marked with asterisks. Inset (a): effect of I h treatment with TFP in darkness. (B) Recovery of actin bundles by continuous wBL $(\mathrm{I} \mathrm{h})$. The irradiation started 15 min after the onset of TFP treatment. The complete AC reconstruction in dark-adapted mesophyll cells pre-treated with TFP for $30 \mathrm{~min}$ and thereafter incubated with $5 \mathrm{mM} \mathrm{Ca}^{2+}$ or $5 \mathrm{mM}$ $\mathrm{Mg}^{2+}$ for $2 \mathrm{~h}$ (C, D, respectively). Scale bars, $10 \mu \mathrm{m}$.

then diluted with $10 \mathrm{mM}$ PIPES. Thus, the treating solutions contained traces of DMSO ranging from 0.03 to $0.21 \%(\mathrm{v} / \mathrm{v})$. These concentrations of DMSO did not affect light-induced chloroplast responses (results not shown). Control experiments were carried out using $10 \mathrm{mM}$ PIPES and, additionally, $5 \mathrm{mM} \mathrm{KNO}_{3}$ (also in PIPES). The latter solution was used because $\mathrm{Ca}^{2+}$ and $\mathrm{Mg}^{2+}$ were applied as nitrates. No significant differences existed between chloroplast responses and/or cytoskeleton images in these two control solutions. Except for PIPES (Duchefa) all chemicals came from Sigma. The solutions were prepared with spectrochemically pure water, and their $\mathrm{pH}$ was adjusted to 6.8 with $\mathrm{NaOH}$. They were stored in calcium-free plastic containers washed with concentrated $\mathrm{HNO}_{3}, 10 \mathrm{mM}$ EGTA and rinsed several times with spectrochemically pure water. Concentrations and incubation times were optimized in preliminary tests for each solution on the basis of clearly observable changes in the actin cytoskeleton and chloroplast movement. The chosen incubation periods ranged from $30 \mathrm{~min}$ to $3 \mathrm{~h}$.

\section{Preparation of samples}

The whole plant was adapted to darkness for at least $12 \mathrm{~h}$ before the experiment. The leaves were detached, the lower epidermis was removed and the tissue was cut into small pieces. After gentle infiltration with distilled water the pieces were stored in water for further usage. During storage, which never exceeded $12 \mathrm{~h}$, no disturbances in the appearance of the AC or chloroplast responses were observed. Several samples at a time were infiltrated with a control or test solution and incubated as required, with constant slow mixing. All infiltrations were done in plastic syringes. Following incubation, some samples were placed on microscope slides to assess the AC organization, while other samples were used for photometric measurements. To prevent drying, the microscope preparations were enclosed in parafilm chambers sealed with silicon grease. All samples were prepared under green safe light and stored in the dark at room temperature.

\section{Confocal Microscopy}

The fluorescence of GFP was visualized with the confocal microscope BioRad MRC 1024 (BioRad, Hercules, CA). Images were collected using a 60× (NA 1.4) PlanApo oilimmersion objective mounted on a Nikon microscope. Fluorescence was excited with blue light at $488 \mathrm{~nm}$ emitted by a $100 \mathrm{~mW}$ argon-ion air-cooled laser (ITL, USA). GFP fluorescence was viewed in the green channel, with the filter 540 DF30, and autofluorescence of chloroplasts - in the red channel, with the filter 585LP. The argon-ion laser was used at $10 \%$ (sporadically at $30 \%$ ) of the maximum power for imaging.

The illumination was performed in the microscope or under a separate halogen lamp. For BL, the lamp was fitted with blue filter foil ( $\lambda \max 424 \mathrm{~nm}$, half-band width, 381-482 nm, Filmfabrik Wolfen, Wolfen, Germany). For $\mathrm{RL}(\lambda \max 646 \mathrm{~nm})$, the lamp was fitted with an RG1 filter, a C805 heat absorbing filter (Schott, Jena, Germany), and a dichroic short-pass filter (PZO, Warszawa, Poland). The applied fluence rates of blue and red light had equivalent quantum fluxes, weak blue (wB) $0.4 \mathrm{Wm}^{-2}$ and weak red (wR) $0.24 \mathrm{Wm}^{-2}$, strong blue (SB) $10 \mathrm{Wm}^{-2}$ and strong red (SR) $6.7 \mathrm{Wm}^{-2}$. The fluence rates were measured with a silicon photodiode calibrated against a LI-COR quantum meter (Li-Cor, Lincoln, NB, USA). Samples were irradiated for $60 \mathrm{~min}$ with weak and $20 \mathrm{~min}$ with strong light in the microscope. The effects of strong light were visible after $20 \mathrm{~min}$; longer irradiation caused fading of fluorescence due to GFP photobleaching. The appearance of AC was checked before and immediately after every irradiation with the confocal microscope. The spongy mesophyll cells situated at least 3 cells away from the vessels were tested. The AC of cells situated near vessels and tracheids was less sensitive to the treatments applied in this study. 

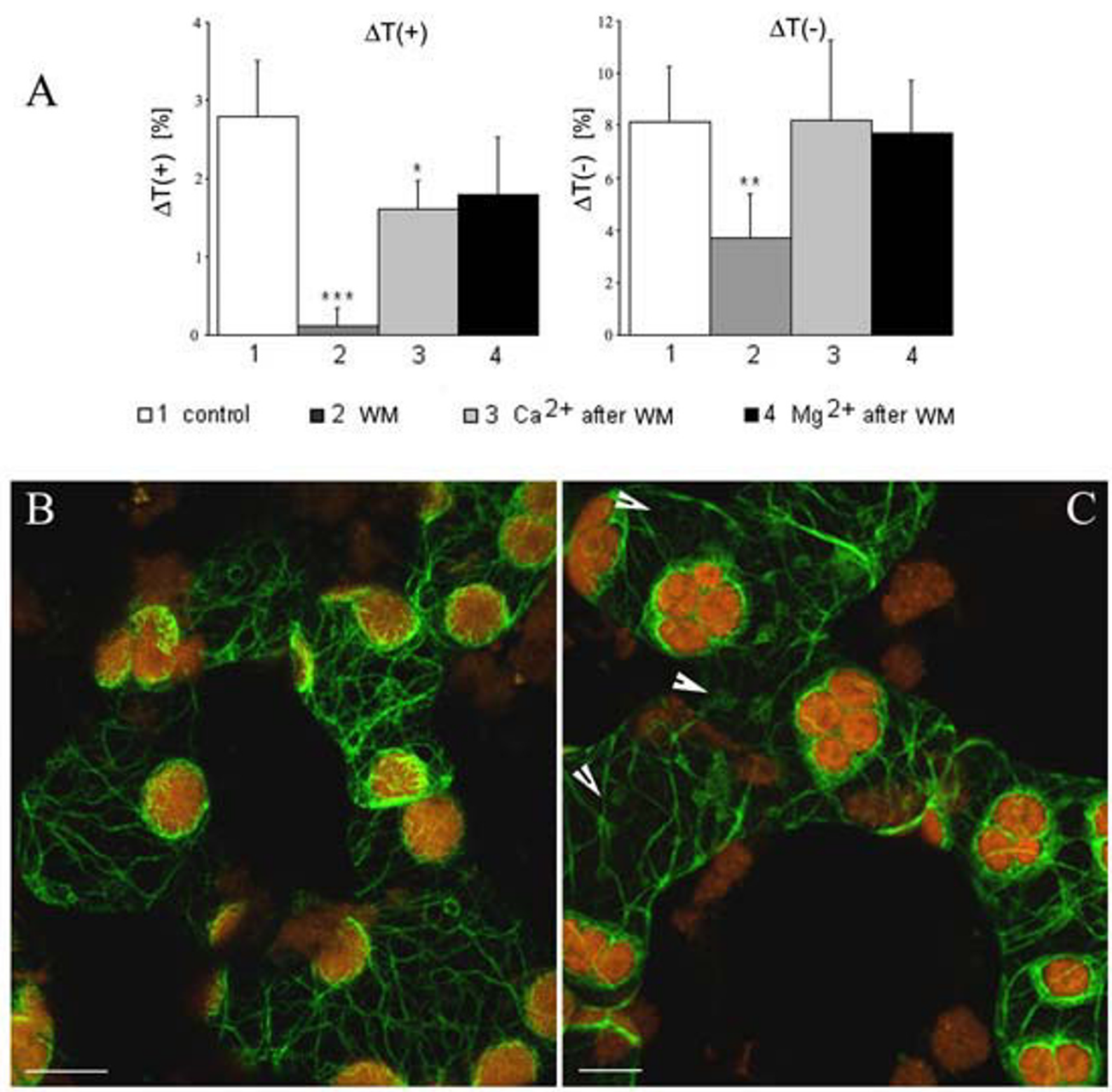

\section{Figure 7}

Effect of wortmannin (WM) on BL-induced chloroplast responses and on the actin cytoskeleton in tobacco leaves. (A) Amplitudes: $\Delta \mathrm{T}(+)$ of weak $\left(\mathrm{wBL}, 0.4 \mathrm{Wm}^{-2}\right)$, and $\Delta \mathrm{T}(-)$ of strong $\left(\mathrm{SBL}, 10 \mathrm{Wm}^{-2}\right)$ blue light-induced responses after $1.5 \mathrm{~h}$ incubation with $10 \mu \mathrm{M}$ WM. The leaves were subsequently treated with $5 \mathrm{mM} \mathrm{Ca}^{2+}$ or $\mathrm{Mg}^{2+}$ for $3 \mathrm{~h}$ (right columns). Averages of 5-7 measurements. Error bars represent SD. Asterisks denote the significance of differences ( $p$-value calculated with the unpaired t-test, ${ }^{*} \mathrm{p}=0,05-0,00 \mathrm{I} ;{ }^{* *} \mathrm{p}=0,00 \mathrm{I}-0,000 \mathrm{I} ;{ }^{* * *} \mathrm{p}<0,000 \mathrm{I}$ ). The network of actin bundles in the mesophyll cells of the transgenic tobacco after I,5 h incubation with $10 \mu \mathrm{M}(\mathrm{B})$, and $50 \mu \mathrm{M}(\mathrm{C}) \mathrm{WM}$. Note the chloroplast clustering and disturbances of $A C$ integrity (arrowheads) at the higher WM concentration. Scale bars, $10 \mu \mathrm{m}$. 
Images were assembled from 3 to 15 optical sections collected with $0.5 \mu \mathrm{m}$ steps. All the images presented show the AC at the periclinal walls (abaxial side) of mesophyll cells. No differences were observed in the structure of the $\mathrm{AC}$ at the adaxial side. Mitochondria were stained by 10 min incubation with TMRE ( $10 \mu \mathrm{M}$, tetramethyl rhodamine ethyl ester, Sigma) and the images were merged with those of GFP.

Each experimental variant was repeated in at least 3 independent series, with 3 to 6 images collected in each series.

\section{Image analysis and processing}

To quantify changes in cytoskeleton architecture thickness of actin bundles in cytoplasm and order parameter of actin baskets at chloroplasts were calculated on single optical sections separately (i.e. 2D methods were used). Sections containing actin-GFP fluorescence and chloroplast autofluorescence were pre-processed using $3 \times 3$ hybrid median filter to suppress noise. Images corresponding to these two bands of fluorescence were segmented to isolate chloroplasts and AC using global thresholding. The threshold levels were calculated using Otsu algorithm [45] and all data from control experiments as an input.

In order to compute thickness of AFs, binary masks corresponding to chloroplasts were dilated (single pass, $3 \times 3$ uniform structuring element) and subtracted from the binary masks corresponding to total actin. The resulting images were convolved with gradient magnitude operator (gaussian derivative, $\sigma=1$ ), thresholded and skeletonized in order to isolate edges. Average thickness of these AFs was calculated (on a section by section basis) by dividing the area (sum of respective cytoplasmic actin binary mask) by total length of edges (sum of respective skeletonized binary image). The thickness was typically greater in the top images of a stack (horizontal sections through actin layer) than in bottom images (vertical sections). Therefore, minimum and maximum thickness of AF section was estimated by taking, respectively, 1st and 99th percentile of data set corresponding to all optical sections (15-30 sections, 3-5 stacks) taken at the same experimental conditions. The maximum corresponded to thickness of AF bundles visible in the maximum z-projections of the confocal stacks (all images shown in this work). The minimum corresponded to the thickness measured in the plane orthogonal to the former. One should note that the minimum was in the range of 0.7 to $1.1 \mu \mathrm{m}$. under all conditions. These facts indicate consistent performance of AF bundle segmentation and measurement routine. Furthermore, one may postulate that the bundles were not circular and that the changes of their architecture were directional.
To quantify the sharpness (energy) of pattern of AF distribution in the baskets around chloroplasts the images corresponding to GFP were convolved with gradient magnitude operator (gaussian derivative, $\sigma=0.75$ ). Average intensity in these images was calculated over areas marked by the binary masks corresponding to chloroplasts. The masks were calculated as described in the previous section, but merged chloroplasts were separated using watershed (performed on original images). The density was computed by taking percentiles (1, 5, 50, 95, $99)$ of the data set corresponding to the same experimental conditions.

\section{Photometric method}

Chloroplast movements were measured with a custommade double-beam photometer [46]. Monochromatic red light $\lambda=660 \mathrm{~nm}$ at a fluence-rate of $0,1 \mu \mathrm{mol} \mathrm{m} \mathrm{m}^{-2} \mathrm{~s}^{-1}(15$ $\mathrm{mWm}^{-2}$ ), modulated with the frequency of $800 \mathrm{~Hz}$ served as the measuring light. Actinic light was obtained from a halogen lamp $(100 \mathrm{~W}, 12 \mathrm{~V})$. BL $\left(\lambda_{\max } 423 \mathrm{~nm}\right.$, half-band width, $86 \mathrm{~nm}$ ) was obtained with a combination of filters, BG12, BG23, GG13 and a heat-absorbing C805 (all from Schott, Jena, Germany). Light-induced chloroplast responses were recorded as changes in light transmission through the leaf pieces.

Only samples with similar levels of initial transmission after dark adaptation (between 38 and 41\%) were selected. The sample was placed on a glass slide in a drop of the incubation medium. It was closed in a miniature chamber formed by a saran-wrap cover stretched over a metal ring mounted on a parafilm seal. The dark-adapted leaf sample was illuminated perpendicular to its adaxial surface with weak blue light $\left(0.4 \mathrm{Wm}^{-2}\right)$ followed by strong blue light $\left(10 \mathrm{Wm}^{-2}\right)$ for 45 minutes with each fluence rate. The following parameters were measured/calculated to characterize the chloroplast responses, 1) amplitudes - transmission changes after 45 min of each response 2) velocities - first derivatives of the initial (lasting about $10 \mathrm{~min}$ ) linear fragments of the respective transmission curves (see Fig. 1A).

\section{Abbreviations}

ABPs: actin binding proteins; AC: actin cytoskeleton; AFs: actin filaments; GFP: green fluorescent protein; $\Delta \mathrm{T}$ : transmission change; TFP: trifluoperazine; TMRE: tetramethyl rodamine ethyl ester; wL: weak light; wBL/wRL: weak blue/red light; SL: strong light; SBL/SRL: strong blue/red light: WM: wortmannin.

\section{Authors' contributions}

AAM created the transgenic plant, designed the study, carried out the experiments and prepared the manuscript. TB carried out the quantitative image analysis. HG conceived 
of the study, supervised the collection, analysis and interpretation of data, and helped in preparing the manuscript. All authors read and approved the final manuscript.

\section{Additional material}

\section{Additional file 1 \\ Energy of actin distribution pattern in F-actin baskets surrounding chloroplasts. Sharpness (energy) of actin distribution pattern in the bas- kets at the chloroplasts in control (Ctrl), and in the presence of $5 \mathrm{mM}$ $\mathrm{Ca}^{+2}$ or $\mathrm{Mg}^{+2}$, in cells treated for $2 \mathrm{~h}$ with the ions only, or pre-treated with $1 \mathrm{mM}$ EGTA or $20 \mu \mathrm{M}$ TFP for $30 \mathrm{~min}$. The network energy was meas- ured in cells adapted to darkness (Dad, gray bars) and in cells illuminated with strong (SB) or weak (wB) blue light (bright and dark blue bars, respectively) and with strong $(S B)$ or weak $(w R)$ red light (bright and dark red bars, respectively). Higher energy corresponds to higher inhomo- geneity of actin structure. The energy was computed in arbitrary units (A.U., see Materials and Methods) as 1st, 50th (median) and 99th per- centile of the data corresponding to a population of chloroplasts in each set of experimental conditions. Error bars represent $95 \%$ confidence intervals. One may note that energy corresponding to lowest (1st) percentile was non-zero and similar in all the conditions. This fact indicates the presence of constitutive non-uniform actin distribution (structure) at the chloro- plasts. The disruption of actin baskets under strong irradiation (red and blue) was manifested clearly only at the highest (99th) percentile of the pattern energy data. Therefore, it may be postulated that filaments (and their edges), which contribute to this effect, occupy only minor fraction of chloroplast surface. \\ Click here for file \\ [http://www.biomedcentral.com/content/supplementary/1471- 2229-9-64-S1.jpeg]}

\section{Additional file 2}

Reorganization of F-actin during strong blue light irradiation. Changes in reorganization of actin bundles and in chloroplast distribution in lower mesophyll cells during SBL irradiation. The first image shows the weak white light-adapted tissue. Images B and C were collected after 10 and $20 \mathrm{~min}$ respectively. The avoidance response of chloroplasts needs approximately $1.5 \mathrm{~h}$ to be completed. Therefore, only partial redistribution of chloroplasts towards profile position is noticeable in several cells (marked with arrows). Scale bar, $10 \mu \mathrm{m}$.

Click here for file

[http://www.biomedcentral.com/content/supplementary/14712229-9-64-S2.jpeg]

\section{Additional file 3}

Reorganization of wide F-actin strands in a tobacco cell adjacent to the vascular bundle. Dynamic reorganization of wide F-actin strands in a tobacco cell adjacent to the vascular bundle irradiated with SBL. Time series collected every $2 \mathrm{~min}$.

Click here for file

[http://www.biomedcentral.com/content/supplementary/14712229-9-64-S3.avi]

\section{Additional file 4}

Reorganization of wide F-actin strands in tobacco mesophyll cells. Time series collected every $2 \mathrm{~min}$.

Click here for file

[http://www.biomedcentral.com/content/supplementary/14712229-9-64-S4.avi]

\section{Additional file 5}

Reorganization of wide F-actin strands in tobacco mesophyll cells. Note the dynamic movement of nucleus in the upper cell. Time series collected every $2 \mathrm{~min}$.

Click here for file

[http://www.biomedcentral.com/content/supplementary/14712229-9-64-S5.avi]

\section{Additional file 6}

Reversibility of the "diffusion" effect induced by strong blue light. Single confocal scans of a cortical part of the same mesophyll cell after consecutive irradiations with continuous SBL $(20 \mathrm{~min})$ and $w B L(60 \mathrm{~min})$. (A) Branched actin network in the dark-adapted cell; (B) Diffuse F-actin forming single widened strands after SBL irradiation (marked with arrow); (C) Reconstruction of distinct bundles (marked with arrows) by $w B L$. Scale bars, $10 \mu \mathrm{m}$.

Click here for file

[http://www.biomedcentral.com/content/supplementary/14712229-9-64-S6.jpeg]

\section{Additional file 7}

The effect of strong light on F-actin in cells with modified $\mathrm{Ca}^{2+} / \mathrm{Mg}^{2+}$ levels. Diffuse widened strands appearing after exposure to continuous strong blue (left panels) and red light (right panels). Prior to irradiation, the samples were incubated with (A) $5 \mathrm{mM} \mathrm{Ca} a^{2+}$ for $2 \mathrm{~h}$, (B) $5 \mathrm{mM} \mathrm{Mg}^{2+}$ for $2 \mathrm{~h},(C, D) 1 \mathrm{mM}$ EGTA for $45 \mathrm{~min},(E, F) 20 \mu \mathrm{M}$ solution of calmodulin inhibitor TFP for $45 \mathrm{~min},(\mathrm{G}) 20 \mu \mathrm{M}$ TFP for 45 min followed by $5 \mathrm{mM} \mathrm{Ca} a^{2+}$ for $2 \mathrm{~h}$, (H) $20 \mu \mathrm{M}$ TFP for $45 \mathrm{~min}$ followed by $5 \mathrm{mM}$ $\mathrm{Mg}^{2+}$ for $2 \mathrm{~h}$. Scale bars, $10 \mu \mathrm{m}$.

Click here for file

[http://www.biomedcentral.com/content/supplementary/14712229-9-64-S7.jpeg]

\section{Additional file 8}

Combined action of EGTA and calcium ionophore on the cytoskeleton. Effect of $1 \mathrm{mM}$ EGTA + calcium ionophore A23187 on the actin cytoskeleton, after 15 (A), 30 (B) and $45 \mathrm{~min}$ (C). Note the transient formation of baskets around the chloroplasts. The filaments making baskets developed slowly over the first $30 \mathrm{~min}$ of the incubation period (b) and gradually faded away over the next $30 \mathrm{~min}$. Scale bars, $10 \mu \mathrm{m}$.

Click here for file

[http://www.biomedcentral.com/content/supplementary/14712229-9-64-S8.jpeg]

\section{Acknowledgements}

The bacteria containing the plastin-GFP construct were kindly provided by Dr A.C.J. Timmers (Institut National de la Recherche Agronomique, Castanet-Tolosan, France). We would like to thank Prof. P. Wojtaszek (Institute of Molecular Biology and Biotechnology, A. Mickiewicz University, Poznań) for his helpful advice and encouragement. We would also like to thank Prof. J. Dobrucki (Division of Cell Biophysics, Jagiellonian University, Kraków) for the use of confocal microscope in his laboratory, for his advice and help. We express our gratitude to Prof. W. Korohoda and to Dr Z. Madeja (Department of Cell Biology, Jagiellonian University, Kraków) for valuable critical comments on this paper.

This study was partly supported by Grants PBZ I I 0/P04/2004 (strong light experiments) and PB I395/B/P0I/2007/33 from Polish Ministry of Science and Higher Education. 


\section{References}

I. Grolig F: Organelle movements: transport and positioning. In The plant cytoskeleton in cell differentiation and development Edited by: Hussey PJ. Oxford, Blackwell Publishing; 2004: I48-I 75.

2. Drøbak BK, Franklin-Tong VE, Staiger CJ: The role of the actin cytoskeleton in plant cell signaling. New Phytologist 2004, 163:13-30.

3. Haupt W, Scheuerlein R: Chloroplast movement. Plant Cell Environ 1990, I3:596-6I4.

4. Sakamoto K, Briggs WR: Cellular and subcellular localization of phototropin I. Plant Cell 2002, I 4:|723-I735.

5. Harada A, Sakai T, Okada K: PhotI and phot2 mediate blue light-induced transient increase in cytosolic $\mathbf{C a}^{2+}$ differently in Arabidopsis leaves. Proc Natl Acad Sci USA 2003, 1 00:8583-8588.

6. Jarillo JA, Gabryś'H, Capel J, Alonso JM, Ecker JR, Cashmore AR: Phototropin-related NPL-I controls chloroplast relocation induced by blue light. Nature 200I, 4 I 0:952-954.

7. Sakai T, Kagawa T, Kasahara M, Swartz TE, Christie JM, Briggs WR, Wada M, Okada K: Arabidopsis nph I and npl I, Blue light receptors that mediate both phototropism and chloroplast relocation. Proc Natl Acad Sci USA 200I, 98:6969-6974.

8. Sato $Y$, Wada M, Kadota A: Choice of tracks, microtubules and or actin filaments for chloroplast photo-movement is differentially controlled by phytochrome and a blue light receptor. J Cell Sci 200I, I | 4:269-279.

9. Takagi S: Actin-based photo-orientation movement of chloroplasts in plant cells. J Exp Biol. 2003, 206( I 2): | 963-1969.

10. Malec P, Rinaldi RA, Gabryś H: Light-induced chloroplast movements in Lemna trisulca. Identification of the motile system. Plant Sci 1996, 120:127-137.

II. Wojtaszek P, Anielska-Mazur A, Gabryś H, Baluška F, Volkmann D: Recruitment of myosin VIII towards plastid surfaces is rootcap specific and provides the evidence for actomyosin involvement in root osmosensing. Funct Plant Biol 2005, 32:721-736.

12. Krzeszowiec W, Gabryś H: Blue light-induced reorganization of myosins in Arabidopsis thaliana. Plant Signal Behav 2007, 2:333-336

13. Kandasamy MRB, Meagher RB: Actin-organelle interaction, association with chloroplast in Arabidopsis leaf mesophyll cells. Cell Motil Cytoskeleton 1999, 44: I I0-1 I8.

14. Gabryś H: Blue light-induced orientation movements of chloroplasts in higher plants. Recent progress in the study of their mechanisms. Acta Physiol Plant 2004, 26:473-478.

I5. Spalding EP, Folta KM: Illuminating topics in plant photobiology. Plant Cell Environ 2005, 28:39-53.

16. Sanders D, Brownlee C, Harper JF: Communicating with calcium. Plant Cell 1999, I I:691-706.

17. Sanders D, Pelloux J, Brownlee C, Harper JF: Calcium at the crossroads of signaling. Plant Cell. 2002, I4(Suppl):S40I-S4I7.

18. Snedden WA, Fromm H: Calmodulin as a versatile calcium signal transducer in plants. New Phytol 200I, I 5 I:35-66.

19. White PJ, Broadley MR: Calcium in plants. Ann Bot (Lond) 2003, 92:487-5II.

20. Baum G, Long JC, Jenkins GI, Trewavas AJ: Stimulation of the blue light phototropic receptor NPHI causes a transient increase in cytosolic Ca ${ }^{2+}$. Proc Natl Acad Sci USA 1999, 96: I 3554-I 3559.

21. Babourina $O, N$ Newman I, Shabala S: Blue light-induced kinetics of $\mathrm{H}^{+}$and $\mathrm{Ca}^{2+}$ fluxes in etiolated wild-type and phototropinmutant Arabidopsis seedlings. Proc Natl Acad Sci USA 2002, 99:2433-2438.

22. Stoelzle S, Kagawa T, Wada M, Hedrich R, Dietrich P: Blue light activates calcium-permeable channels in Arabidopsis mesophyll cells via phototropin signaling pathway. Proc Natl Acad Sci USA 2003, 100:|456-|46|.

23. Takagi S: Photoregulation of cytoplasmic streaming, Cell biological dissection of signal transduction pathway. J Plant Res 1997, I 1 0:299-303.

24. Tlałka M, Gabryś H: Influence of calcium on blue-light-induced chloroplast movement in Lemna trisulca L. Planta 1993, | 89:49|-498.

25. Tlałka $M$, Fricker $M$ : The role of calcium in blue-light-dependent chloroplast movement in Lemna trisulca L. Plant J 1999 , 20:46I-473.

26. Grabalska M, Malec P: Blue light-induced chloroplast reorientations in Lemna trisulca L. (duckweed) are controlled by two separable cellular mechanisms as suggested by diffrent sensitivity to wortmannin. Photochem Photobiol 2004, 79:343-348.

27. Krzeszowiec W, Rajwa B, Dobrucki J, Gabryś H: Actin cytoskeleton in Arabidopsis thaliana under blue and red light. Biol Cell 2007, 99:25I-260.

28. Timmers ACJ, Niebel A, Balague C, Dagkesamanskaya A: Differential localisation of GFP fusions to cytoskeleton-binding proteins in animal, plant, and yeast cells. Protoplasma 2002, 220:69-78.

29. Zurzycki J: Studies on the centrifugation of chloroplasts in Lemna trisulca. Acta Soc Bot Pol 1960, 29:385-393.

30. Kadota $A$, Wada $M$ : Photoinduction of formation of circular structures by microfilaments on chloroplasts during intracellular orientation in protonemal cells of the fern Adiantumcapillus-veneris. Protoplasma 1992, 167:97-107.

31. Augustynowicz J, Lekka M, Burda K, Gabryś H: Correlation between chloroplast motility and elastic properties of tobacco mesophyll protoplasts. Acta Physiol Plant 200I, 23:291-302.

32. Kengen HMP, de Graaf $\mathrm{BH}$ : Microtubules and actin filaments co-localize extensively in non-fixed cells of tobacco. Protoplasma 1991, I63(I):62-65

33. Snowman BN, Kovar DR, Shevchenko G, Franklin-Tong VE, Staiger C]: Signal-mediated depolymerization of actin in pollen during the self-incompatibility response. Plant Cell 2002, | 4:2613-2626.

34. Frieden C: Polymerization of actin, Mechanism of the $\mathbf{M g}^{2+}$ induced process at $\mathrm{pH} 8$ and $20^{\circ} \mathrm{C}$. Proc Natl Acad Sci USA 1983 80:65।3-65।7.

35. Carlier MF, Pantaloni D, Korn ED: The effects of $\mathbf{M g}^{\mathbf{2 +}}$ at the highaffinity and low affinity sites on the polymerization of actin and associated ATP hydrolysis. I Biol Chem 1986, 26 I: : 0785-10792.

36. Carlier MF: Actin polymerization and ATP hydrolysis. Adv Bioph 1990, 26:5I-73.

37. Estes JE, Selden LA, Kinosian HJ, Gershman LC: Tightly-bound divalent cation of actin. I Muscle Res Cell Motil 1992, I3:272-284.

38. Strzelecka-Gołaszewska H, Woźniak A, Hult T, Lindberg U: Effects of the type of divalent cation, $\mathrm{Ca}^{2+}$ or $\mathrm{Mg}^{2+}$, bound at the high affinity site and of the ionic composition of the solution on the structure of F-actin. Biochem J 1996, 3 I 6:713-721.

39. Kadota A, Wada M: Photoorientation of chloroplasts in protonemal cells of the fern Adiantum as analized by use of a video-tracking system. J Plant Res 1992, I 05:265-279.

40. Shabala S, Hariadi Y: Effect of magnesium availability on the activity of plasma membrane ion transporters and lightinduced responses from broad bean leaf mesophyll. Planta 2005, $221: 56-65$

41. Murphy E: Mysteries of magnesium homeostasis. Circ Res 2000, 86(3):245-8.

42. Shaul $O$ : Magnesium transport and function in plants, the tip of the iceberg. BioMetals 2002, I 5:309-323.

43. Gardner RC: Genes for magnesium transport. Curr Opin Plant Biol 2003, 6:263-267.

44. Jung JY, Kim YW, Kwak JM, Hwang JU, Young Y, Schroeder JI, Hwang I, Lee Y: Phosphatidylinositol 3- and 4-phosphate are required for normal stomatal movements. Plant Cell 2002, I 4:2399-24I 2.

45. Otsu N: A threshold selection method from gray-scale histogram. IEEE Trans. Systems, Man, and Cybernetics 1979, 9:62-66.

46. Walczak T, Gabryś H: New type of photometer for measurements of transmission changes corresponding to chloroplast movements in leaves. Photosynthetica 1980, 14:65-72. 